\title{
Evaluación de procesos cognitivos y optimización instruccional
}

\author{
CÀndid Genovard, Concepció Gotzens, Antoni Castelló, \\ Carla González y Juan Pablo González \\ Universidad Autónoma de Barcelona
}

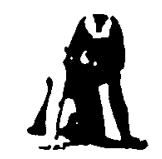

\section{Resumen}

Las cuatro lineas de investigación que se presentan sobre evaluación de la docencia experta, aptitudes cognitivas en entomos de alta tecnologia, identificación de alumnos superdotados y con talento y aptitudes en la educación artística, representan dimensiones teórico-descriptivos (las dos primeras) y descriptivo-aplitadas (las dos últimas) que se inscriben en un enfoque en el que se resalta la evaluación de variables intervinientes en el proceso instruccional, desde una conceptualización cognitiva, coma vía para lograr la optimización de los propios procesos instruccionales

Palabras clave: Evaluación, Procesos cognitivos, Instrucción.

\section{Evaluation of cognitive processes and instructional optimization}

\section{Abstract}

Four lines of research are discussed: assessment of expert teaching; cognitive abilities in bigh-technology environments; identification of gifted and talented students; and teacher's ability to understand students' aestbetic aptitude in art instruction. The first two represent theoretical-descriptive lines of research and the latter two represent descriptive-applied research. These four areas share a common approach to research which underlines the assessment of intervening variables in the instructional process - from a cognitive conceptualization - as a means to achieve the optimization of the in. structional processes.

Keywords: Assessment, Cognitive processes, Instruction.

Dirección de los autores: Facultad de Psicología, Universidad Autónoma de Barcelona, Bellaterra, 08193 Barcelona. 


\section{INTRODUCCION}

La línea de trabajo que presentamos se desarrolla a partir de dos coordenadas principales que confluyen en un objetivo común: de un lado, la evaluación de elementos implicados en el desarrollo y ejecución de actividades y procesos instruccionales y de otro, el análisis de estos elementos desde una perspectiva básicamente cognitiva, siendo el propósito común mencionado el de conseguir una mejora u optimización de los procesos de enseñanza-aprendizaje.

Tanto la psicología de la educación como la psicología de la instrucción incorporan en su propia definición el objetivo mencionado de mejorar los procesos a cuyo estudio se dedican, lo que las convierte en disciplinas aplicadas (Gage \& Berliner, 1984; Genovard \& Gotzens, 1990). En este sentido nuestro móvil de estudio se sitúa plenamente en el ámbito psicoeducativo y psicoinstruccional. Sin embargo la línea de investigación que presentamos supone una opción específica dentro del propósito general de incidir, mejorando, en los procesos de enseñanza-aprendizaje; dicha opción se refiere al conocimiento de algunas variables intervinientes en dicho proceso, su explicación en términos cognitivos y su control a través de mecanismos de evaluación.

Efectivamente, todas las variables se examinan desde un marco de referencia básicamente cognitivo, entendiendo por tal aquél que es capaz de procesar la información que le llega del entorno e interactuar con ella; no se trata pues del análisis de sistemas cerrados de procesamiento de información, sino de variables que adquieren sentido por la contribución que hacen al proceso instruccional en términos de procesamiento activo, e interactivo, de la información (Klausmeier, 1985) y de una respuesta al entorno al que, a su vez, aporta nuevos datos e informaciones, estableciéndose así una suerte de intercambio que no hace sino poner de manifiesto el carácter esencialmente dinámico e interactivo del proceso instruccional.

Sin duda se trata de una opción no exenta de riesgos y complejidades, sin embargo y en contrapartida es rica por cuanto es capaz de generar e integrar estudios diversos y se apoya sobre una base teórica suficientemente sólida y reconocida en el ámbito científico psicoeducativo internacional.

El análisis cognitivo se realiza sobre dos de las variables fundamentales del campo instruccional, como son el docente y el alumno (Genovard, Gotzens \& Montane, 1987). Dicho análisis aporta una serie de elementos referidos a los procesos internos, informaciones relevantes y estructuras implicadas, los cuales inciden explícitamente en las interacciones instruccionales, permitiendo la explicación y predicción de importantes aspectos de las mismas. Por otra parte este análisis resulta igualmente relevante para la definición y delimitación del objeto de medida y su posterior evaluación (entendida como medición más interpretación).

Tal como se ha apuntado, la finalidad de esta línea de investigación radica en la mejora u optimización de los procesos instruccionales, por lo que los procesos cognitivos evaluados son estrictamente aquéllos que pueden desempeñar algún papel en el entorno instruccional. De alguna forma, el análisis de procesos cognitivos resulta en una ampliación del ámbito habitual de investigación, al menos en dos sentidos: en primer lugar, aportando una serie de elementos explicativos de algunos fenómenos e interacciones netamente instruccionales, difícilmente abordables desde el ámbito estricto de la Psicología de la Instrucción; así, la consideración de profesor y alumno como procesadores de información interactivos conlleva la profundización en los procesos realizados por los mismos, como importantes condicionantes -aunque, en muchos casos, no como únicos determinantes- de las interacciones entre los mismos o entre éstos y las otras grandes variables. En segundo lugar, el conocimiento y medición de los procesos y contenidos cognitivos incide en la mayor predictibilidad de una parte importante de comportamientos -aprendizaje, interacciones instruccionales o estrategias de enseñanza, entre otros- de alta relevancia instruccional (Klausmeier, 1985); obviamente, este conocimiento y posibilidad de predic- 
ción es la base sobre la que se sustentará toda intervención y control sobre los comportamientos citados, redundando en la optimización de los procesos de enseñanzaaprendizaje.

Así, por ejemplo, la evaluación de la docencia constituye una pieza altamente significativa del engranaje instruccional, en el sentido de que la forma en que se lleve a cabo la docencia afectará indudablemente el desarrollo global del proceso (Berliner, 1989). En este caso, la evaluación del comportamiento docente, investigación a la que nos referiremos más adelante, se enmarca en la línea de investigación que aquí presentamos, siendo su objetivo básico el mejoramiento del proceso instruccional. El comportamiento del profesor se conceptualiza en términos cognitivos cuyos correlatos conductuales pueden ser objeto de observación directa, pero cuya interpretación cae obviamente en el ámbito de la inferencia de sus propósitos, intenciones, motivos y justificaciones. Por ello y partiendo de la elaboración de un modelo de actividad cognitiva explicativa del comportamiento docente se evalúa la calidad del mismo, atendiendo al tipo de procesos cognitivos que lleva a cabo, los cuales no se hallan en un sistema cerrado de procesamiento de información, sino que se alimentan y confrontan con los datos del entorno en el que se lleva a cabo la docencia, siendo la capacidad de interactuar con este entorno una exigencia del propio proceso, como un índice de calidad del mismo (Genovard et al., en prensa).

En este sentido, la importancia de los procesos cognitivos para actividades genuinamente instruccionales quedaría reflejada en, por ejemplo, la captación por parte del profesor de índices significativos del nivel de aprovechamiento del alumno de la docencia impartida en base a pistas o señales concretas observables en el comportamiento de los alumnos (Berliner, 1987a). Esta conducta es, a nuestro juicio, tanto un exponente del carácter genuinamente interactivo del comportamiento docente con el entorno en el que se desarrolla, como un índice de la calidad de dicho comportamiento, siendo más positivo cuanto mayor sea la sensibilidad que muestre el profesor hacia estas señales - las que aportan información significativa a nivel instruccional - así como su capacidad de interpretarlas y reajustar su respuesta instruccional en función de las mismas y de los propósitos establecidos para el desarrollo del proceso en cuestión.

Esta línea de trabajo incluye diversas investigaciones que comparten tanto el objetivo de optimización instruccional, como el sistema o procedimiento seguido (evaluación de procesos) para su desarrollo. Lo que varía, dando así lugar a diversas investigaciones, es la variable o variables objeto de estudio, las cuales se pueden desglosar en dos elementos diferenciados: el tipo de interacción o interacciones y los procesos cognitivos involucrados en las mismas.

A otro nivel, y en el marco del dualismo evaluación-investigación, cabe señalar que la línea de inivestigación presentada puede calificarse de investigación dirigida a la evaluación, por cuanto el propósito que la guía no es meramente el interés por conocer el tipo de relaciones que se establecen entre diversas variables instruccionales o entre éstas y los procesos cognitivos implicados -interés, por otra parte, perfectamente lícito- sino que es determinar el tipo de valores o variaciones que pueden dar como resultado una propuesta instruccional mejor.

En cuanto al marco teórico de referencia utilizado ya hemos indicado que se asienta en la psicología cognitiva y, dentro de ésta, fundamentalmente en una perspectiva de procesamiento de la información. Sin embargo, las aportaciones teóricas concretas dependen en cada caso de las variedades de cuya evaluación nos ocupamos. Básicamente los estudios desarrollados en esta línea de investigación son cuatro: 1) Superdotación y talento; 2) Educación artística; 3)La docencia experta; y 4) Aptitudes cognitivas implicadas en entornos de alta tecnología. Como ya hemos indicado, todos guardan en común el interés por optimizar la instrucción a partir de la evolución de procesos y variables comprometidos en ella, pero se diferencian notablemente por el tipo de procesos y variables sobre 
los que centramos nuestra atención. Ello ha hecho posible el desarrollo de una línea de investigación coordinada a la vez que diversificada, así como el trabajo en equipo de intereses específicos diferenciados.

Una posible organización conjunta de estos cuatro estudios podría estructurarse a partir de las dimensiones sujeto (de quien se analizan las actividades cognitivas) que puede ser profesor o alumno y nivel de generalidad, refiriéndonos con este término a la polarización hacia el extremo más descriptivo y explicativo de situaciones instruccionales generales o hacia el extremo de aplicación y optimización de situaciones concretas. Estos estudios quedan representados en la figura 1 a partir de los citados criterios.

La segunda de las dimensiones debe ser entendida dentro del ámbito de la Psicología de la Educación, en el sentido en que una mayor generalidad o aproximación hacia el polo teórico no implica el trabajo en el contexto de máxima abstracción o de procesos bá- sicos, sino en aquellos aspectos que siendo, como la propia disciplina, aplicados, se encuentran a un paso de la intervención. En este sentido, diríamos que los resultados de dichos estudios son fundamentalmente descriptivos y explicativos de interacciones instruccionales generales, por lo que consideramos que se sitúan en el polo más teórico de una ciencia aplicada como la Psicología de la Instrucción.

En las siguientes páginas pasamos a describir con mayor detalle cada uno de los estudios que hemos introducido hasta aquí.

\section{SUPERDOTACION Y TALENTO}

Utilizando un criterio cronológico, la primera investigación que cabe citar es la que trata de la evaluación e identificación de individuos superdotados y con talento, con el propósito de aportar consideraciones relativas a la intervención instruccional con estos alumnos (Genovard, 1982, 1983a，1983B; Castello, 1986).

Figura 1

\begin{tabular}{|c|c|c|c|}
\hline & & Polo teórico & Polo aplicado \\
\hline & & \multicolumn{2}{|c|}{ Nivel de generalidad } \\
\hline & & $\begin{array}{l}\text { Descriptivo } \\
\text { Explicativo }\end{array}$ & $\begin{array}{l}\text { Aplicación } \\
\text { Optimización }\end{array}$ \\
\hline \multirow{2}{*}{. $\frac{\stackrel{U}{J}}{\frac{3}{7}}$} & 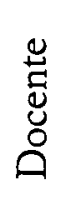 & $\underset{\substack{\text { (alumno) } \\
\text { (contenidos) } \\
\text { (entorno) }}}{\mathrm{DE}}$ & $\begin{array}{l}\mathrm{S} / \mathrm{T} \\
\text { (alumno) }\end{array}$ \\
\hline & 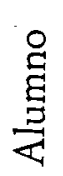 & $\begin{array}{c}\text { HI } \\
\text { (contenidos) } \\
\text { (entorno) }\end{array}$ & EdA \\
\hline
\end{tabular}


Los inicios de esta investigación en nuestro equipo se sitúan a comienzos de los años $80 \mathrm{y}$, aún siendo abundantes, las investigaciones que le sirvieron de base eran eminentemente descriptivas y conceptualmente basadas en las teorías monolíticas de la inteligencia y más específicamente, en la tradición de los estudios de Terman (1925) sobre individuos superdotados, en los que el concepto de CI marcaba el criterio definitivo para establecer la superdotación de los individuos. En otras palabras, los superdotados eran individuos con inteligencia superior lo que, en esta conceptualización, es sinónimo de poseer un CI significativamente alto, o si se prefiere, en términos más actuales, son individuos capaces de ejecutar de forma superior a la habitual el tipo de operaciones intelectuales a partir de las cuales se determina el CI de los individuos.

Las propuestas de autores como Renzulli (1984) que aportan explicaciones alternativas y más ricas al tema de la superdotación, si bien todavía no pueden competir a nivel cuantitativo con la abundante literatura existente en otros países y las consiguientes investigaciones inspiradas en ella, que en gran parte sigue sin despegarse del concepto monolítico de inteligencia humana, representa un avance significativo y sugerente a nivel cualitativo y su resonancia en este ámbito va en progreșo.

Nuestro punto de partida en este tema se fundamenta en la idea de que los alumnos superdotados no sólo ejecutan operaciones intelectuales de forma más perfeccionada que los sujetos no superdotados, sino que desarrollan un funcionamiento intelectual peculiar, por lo que precisan cierto tipo de intervención instruccional diferenciada en relación a los alumnos que no lo son, o no poseen estas capacidades, al igual que la necesita todo alumno que posee características excepcionales, es decir fuera de lo habitual. En otras palabras, los alumnos superdotados son alumnos con necesidades educativas especiales (Castello, 1987).

Inspirándonos en parte en las aportaciones de Renzulli (1984) con el propósito de desmarcarnos, aunque sólo hasta cierto punto, puesto que por el momento no disponemos de la alternativa adecuada, de la máxima según la cual el superdotado es el individuo que posee un CI significativamente superior al resto de la población, llevamos a cabo un estudio de las características de los escolares de 11 años de algunas poblaciones de Cataluña y Baleares, a fin de conocer aspectos relativos a su comportamiento cognitivo y social (Genovard, 1990). Este estudio nos permitió identificar alumnos que, de hecho, más que superdotados, poseían un talento académico destacado, por lo que sus características cognitivas y sociales eran las que se precisan para dar un rendimiento académico superior.

Ello nos condujo a un replanteamiento del tema, que en términos generales se aleja de la mayoría de investigaciones desarrolladas en otros países en esta línea y que nos sitúa en lo que a nuestro juicio es o debe ser el punto de partida del tema de la superdotación: el estudio de la inteligencia humana, cuyo enfoque monolítico no parece ser el más adecuado y satisfactorio para la explicación convincente del comportamiento inteligente humano. La consideración de teorías factoriales $\mathrm{y}$, aún más, de explicaciones capaces de interpretar el funcionamiento inteligente no sólo en términos de diferencias cuantitativas ya sean referidas a una inteligencia general o a factores diversos, sino en términos cualitativos relativos a funciones diferenciadas del intelecto, no parece poder obviarse por más tiempo (Genovard \& Castello, 1990).

A pesar de ello, a nivel de aplicación al que corresponde la realidad instruccional a la que cotidianamente se enfrentan profesores $y$ alumnos, y probablemente por haber sabido ofrecer una fórmula sencilla y económica de expresión del funcionamiento inteligente de los alumnos, el CI continua siendo no sólo un criterio, sino a efectos prácticos, una explicación suficientemente convincente para la toma de decisiones relativas a la vida instruccional del alumno. Así por ejemplo, se acepta que los alumnos con CI bajo precisan acudir al aula de educación especial, o que los alumnos con CI superior pueden optar por la vía de la aceleración académica, etc.

En el momento actual nuestra investiga- 
ción sobre el tema se desarrolla a distintos niveles:

Por una parte, y como ya hemos señalado, llevamos a cabo una aproximación teórica al tema, abordando la conceptualización tanto de la inteligencia, como de la denominada excepcionalidad intelectual, proponiendo un modelo de funcionamiento de la inteligencia basado en el paradigma de procesamiento de la información (Genovard \& Castello, 1990).

Por otra parte, estamos trabajando en la evaluación de las características de alumnos de 5 y 7 años especialmente dotados, con el propósito de detectar los rasgos propios de individuos que poseen otros talentos distintos del académico y con ello, tratar de evitar el sesgo, por demás habitual en los entornos escolares, de que los únicos individuos con posibilidades de recibir algún tipo de atención especial a causa de un desempeño intelectual brillante, son los denominados talentosos académicos, ignorándose al resto de alumnos cuyas dotes no coincidan con lo que se espera observar en un alumno superdotado (buenos rendimientos en materias académicas o en todo caso relativos a las demandas escolares).

En nuestra opinión la tarea de detección de alumnos superdotados o con talento que de forma espontánea realiza el maestro, debe mejorarse y matizarse en el sentido de que sea capaz de reconocer e identificar a todos aquellos que, dadas sus capacidades a nivel intelectual, estén o no directamente relacionadas con los rendimientos escolares, puedan beneficiarse de una intervención especial a nivel educativo.

\section{LA DOCENCIA EXPERTA}

La segunda investigación integrada en la línea que presentamos trata de la evaluación de la docencia experta en el ámbito universitario. La evaluación del comportamiento docente es un tema tan controvertido como importante en el panorama psicoinstruccional. Son numerosos los autores que coinciden en afirmar que la calidad de la docencia es la clave que explica el éxito de los procesos educativos.
Sin entrar en el análisis de las interpretaciones a menudo erróneas a que lleva este tipo de aseveraciones, en el sentido de que tienden a atribuir exclusivamente a la variable docencia la responsabilidad del éxito educativo, resulta obvio que el profesor, en tanto que elemento integrante del proceso instruccional, desempeña un papel relevante en el desarrollo de dicho proceso y que su comportamiento como enseñante, dirigido a potenciar y facilitar el desarrollo y construcción de aprendizajes en los alumnos, ejerce un impacto innegable en la consecución de estos propósitos tanto en términos cuantitativos como cualitativos (Genovard \& Gotzens, 1990).

Nuevamente nos hallamos en presencia de una variable instruccional (comportamiento docente) de cuya evaluación pretendemos derivar una serie de consideraciones que permiten optimizar los procesos de enseñanzaaprendizaje.

En este contexto, la primera cuestión a plantearnos se centró en la conceptualización de lo que debe ser considerado como comportamiento docente de calidad a partir del cual valorar las ejecuciones concretas que puedan observarse en los profesores cuya docencia es evaluada.

Tras una revisión exhaustiva de los criterios utilizados en anteriores investigaciones sobre la evaluación de la docencia (Centra, 1983; Berliner, 1987b; Barnes, 1987; Fernández Sánchez, 1988; Salvador \& Sanz, 1988, entre otros), decidimos desestimar los criterios más frecuentemente utilizados (rendimiento del alumno, experiencia del profesor, etc.) por considerarlos poco aptos para estimar la calidad del comportamiento docente. Frente a ellos, optamos por tomar como marco de referencia los estudios sobre comportamiento experto (Chi, Glaser \& Farr, 1988) y más concretamente sobre comportamiento docente experto (Berliner, 1986, 1987a, 1990).

Así pues, nuestra investigación sobre evaluación de la docencia, concretamente a nivel universitario, asume que el criterio a utilizar para decidir sobre la calidad del comportamiento docente viene definido por el tipo de ejecución que caracteriza al experto. 
A pesar de que actualmente la literatura sobre comportamiento experto se halla en fase expansiva, en el momento de iniciar nuestra investigación carecíamos de un modelo explicativo de dicho comportamiento, por lo que a partir de las características más representativas del docente experto recogidas en diversos estudios, especialmente en los desarrollados por Berliner (1986, 1987a, 1989), elaboramos un modelo en el que básicamente se sintetiza la actividad cognitiva del profesor experto, la cual aporta la explicación procesual a dichas características y comportamientos. Este modelo puede incluirse en el paradigma de procesamiento de información, destacando como rasgo más significativo el hecho de que el comportamiento docente experto se conceptualiza no sólo en términos de macroprocesos cognitivos determinados, sino como el producto de llevar a cabo estos macroprocesos en interacción constante con el entorno instruccional (Genovard et al., en prensa).

Al hilo de lo que ya comentábamos en la introducción de esta presentación, el comportamiento docente experto será aquel capaz de determinar, de la forma más rápida y certera posible, el tipo de destrezas instruccionales que es aconsejable emplear en una situación instruccional determinada, en el bien entendido de que esta decisión será correcta sólo si se capta adecuadamente el tipo de señales instruccionales significativas observadas en la población de alumnos a los que en aquel momento se dirige. Asímismo, la capacidad de reajustar y adaptar las citadas destrezas en función del feed-back explícito o encubierto ofrecido por los alumnos, constituye otro rasgo cognitivo-interactivo característico del profesor experto.

El modelo construido sirvió de base para la elaboración de un instrumento, concretamente un cuestionario dirigido al profesor, que permite la valoración de las capacidades propias de la docencia experta o de calidad.

La realización de una investigación llevada a cabo con una extensa muestra de profesores de la Universidad Autónoma de Barcelona en la que se utilizó, entre otros, el mencionado cuestionario, puso de manifiesto la existencia de diversos perfiles de comporta- miento docente (expertos, profesores con experiencia, no expertos) cada uno de los cuales mostró rendimientos distintos en las capacidades y procesos cognitivos medidos a través del cuestionario (Genovard et al., en - prensa).

Una vez definidos estos perfiles y su composición en términos de actuación cognitiva y tras la administración del cuestionario, es factible determinar cuáles son los procesos cognitivos que están presentes o ausentes y en qué medida en el docente evaluado, proporcionando así orientación sobre el tipo de capacidades y procesos que precisan de un mayor desarrollo o potenciación. Obviamente, algunos de estos procesos no son adquiribles o entrenables, pues dependen de las características en mayor o menor grado innatas del profesor o, en cualquier caso, no susceptibles de modificación en el momento evolutivo del mismo. Sin embargo, determinadas informaciones, o formas de organización de las mismas, pueden ser adquiridas por el profesor, del mismo modo que circuitos cognitivos críticos para la actividad docente eficiente pueden ser entrenados y activados. En suma, la calidad de la docencia es susceptible de mejora, en la mayoría de los casos, aunque no se puede garantizar que todo profesor devenga un experto a partir de dicha intervención. Esta última afirmación ya había sido puesta de manifiesto por aquellos autores que abordaron la temática del profesor experto desde un ámbito estrictamente instruccional. Pero el análisis cognitivo con el que hemos com. plementado dicha temática ha permitido ir más allá, precisando qué procesos son mutables o qué informaciones y formas de estructuración son relevantes.

Una vez más y siempre dentro de la línea de investigación que presentamos, nos ocupamos de evaluar una variable instruccional conceptualizada en términos cognitivos, a fin de sugerir la introducción de mejoras que redunden en la optimización del proceso instruccional global. Tal como se comentaba en la introducción, este enfoque, ha ampliado la descripción del fenómeno y permitido una explicación más completa - y compleja- del mismo. 


\section{EDUCACION ARTISTICA}

La tercera investigación que incluimos se halla todavía en una fase incipiente de su desarrollo y se relaciona con el tema de la educación artística. Este tema, que la Reforma del Sistema Educativo en nuestro país parece querer potenciar tras el estado de olvido absoluto al que se ha visto relegado en nuestro curricula escolares, ha sido y es objeto de vivo interés en otros países en los que se trabaja tanto desde dentro como desde fuera de los respectivos sistemas educativos formales (Eisner, 1986).

Nuestro interés al respecto en el momento presente se centra específicamente en la valoración de materiales ya elaborados que incluyen texto e imágenes, dirigidos al ámbito escolar con el propósito de divulgar y facilitar información sobre temas artísticos monográficos relativos a artes visuales.

En este caso la variable objeto de la evaluación son los materiales confeccionados por una institución de carácter cultural. Nuestros objetivos al respecto se dirigen a:

a) Determinar si dichos materiales sirven a los objetivos mencionados de divulgación e información de temas artísticos entre la población escolar y, más concretamente, a qué niveles de la misma.

b) Determinar si el texto utilizado, tanto a nivel de comprensión e información previa necesaria para su correcta asimilación, como de composición y disposición en la ficha, sirve a los propósitos citados (PARSONS, 1987).

c) Determinar si las imágenes utilizadas y su disposición en el texto son igualmente adecuadas para la consecución de los objetivos propuestos.

d) Establecer las posibles relaciones entre las posibilidades de aprendizaje facilitadas en el material evaluado y las que proporcionan los materiales e instrumentos que el entorno instruccional formal facilita al alumno (GEHLBACH, 1990)

e) $\mathrm{Y}$ finalmente, determinar qué modificaciones deben introducirse a nivel de información verbal e icónica, a fin de que el material cubra los objetivos propuestos.

\section{APTITUDES COGNITIVAS IMPLICADAS EN ENTORNOS DE ALTA TECNOLOGIA}

En este caso se trata de un estudio marco que se dirige a la conceptualización y análisis de los procesos implicados en las interacciones con los entornos high tech. Se deben remarcar respecto a esta investigación diversos aspectos:

a) Los entornos de alta tecnología representan un campo en alza, por la progresiva implantación de los mismos en la vida cotidiana, laboral y educativa (MAYER, 1981). En este sentido, su importancia social, y consecuencias curriculares, es cada vez mayor.

b) Su naturaleza es doble: por una parte, constituyen un entorno, es decir, forman parte de la variable aula/ambiente, modificando su configuración y características tradicionales; por otra son un contenido, en el sentido de que precisan una instrucción específica para su utilización competente.

c) Los entornos de alta tecnología constituyen un contenido de tipo instrumental, diferenciándose de otros contenidos igualmente instrumentales -como la matemática o el lenguaje- en el sentido en que es un instrumento físico y, por tanto, no se utiliza dentro del sistema cognitivo, sino que es externo al mismo. Por otra parte, puede actuar como instrumento de propósito general (representado típicamente por los ordenadores) o específico (como los instrumentos electrónicos o mecánicos diseñados para activiades concretas) compartiendo, pues, las propiedades funcionalmente más relevantes de los aprendizajes instrumentales clásicos.

d) Como consecuencia de lo anterior, se pueden enmarcar en un contexto de interacciones complejas, pues la interacción entre alumno y alta tecnología actúa como medio de interacción entre éstos y un entorno u objetivo externo.

Esta naturaleza compleja pero absolutamente rica que los constituye como transmisores de información, mediadores de la acción, complemento o apéndice cognitivo y materia u objeto de aprendizaje, avala su interés instruccional, no sólo por sus implica- 
ciones genéricas, ya comentadas, sino por su potencial en el contexto psicoeducativo.

$\mathrm{Al}$ igual que en los tres estudios citados hasta aquí, esta investigación aborda los procesos cognitivos, en este caso relacionados con las interacciones con los instrumentos tecnológicos y, a partir de estas, con otros entornos u objetivos.

Este tipo de análisis se centra tanto en los procesos o circuitos cognitivos y sus componentes, como en los contenidos de diversa índole necesarios para su funcionamiento. Cabe destacar que esta aproximación defiere de las anteriores en cuanto las operaciones cognitivas se contemplan desde el binomio hombre-máquina, es decir, el instrumento tecnológico actúa como apéndice o complemento de la mente en las tareas de procesamiento de información.

En términos instruccionales, se encuentran implicados los siguientes aspectos o variables:

a) La alta tecnología como objeto de aprendizaje, es decir, como contenido. En este punto se consideran los elementos que deben ser aprendidos sobre el instrumento y las interacciones con el mismo para su eficiente utilización como tal instrumento. Obviamente, y como ha sido dicho, se trata de un aprendizaje instrumental y nos resultan de especial interés los requisitos y procesos cognitivos necesarios para llevarlo a cabo.

b) La alta tecnología como entorno o con- texto de enseñanza y aprendizaje, es decir como medio para la realización de aprendizajes, sea de contenidos o de procedimientos, o como recurso educativo instrumental.

c) La alta tecnología como ampliación de la variable alumno, en el sentido de apéndice o complemento cognitivo. Por consiguiente las interacciones con el resto de grandes variables instruccionales (docente, contenido y entorno) deben ser conceptualizadas en términos de relación con el binomio alumnotecnología.

En definitiva, presentamos una línea de investigación cuyas características básicas se resumen en los siguientes puntos:

- se resalta el valor de la evaluación de distintas fases y componentes del proceso instruccional.

- Las variables que son objeto de evaluación se conceptualizan en términos cognitivos, con el propósito de establecer no sólo su carácter significativo en el contexto de la situación instruccional, sino también de interpretar a través de los procesos y capacidades descritas, los mecanismos capaces de explicar la interacción que se produce entre los elementos instruccionales.

-El propósito básico que guía esta línea de trabajo consiste en proporcionar información sobre cómo mejorar los procesos instruccionales.

\section{Referencias}

Barnes, J. (1987). Teaching experience. En Dunkin, M. J. (Ed.). The International Enciclopedia of Teaching and Teacher Education. New York: Pergamon.

BerLINER, D. C. (1986). In pursuit of the expert pedagogue. Educational Reserarcher, 5(13), 2-4

BerLINER, D. C. (1987 A). Expert and novice interpretation of classroom data. Washington: Paper presentado en la Convención anual de la American Educational Research Association.

BerLINER, D. C. (1987B). Simple views of teaching and a simple theory of classroom instruction. En Berliner, D.C. \& Rosenshine, B. (Eds.). Talks to teachers. New York: Random House.

BerLINER, D. C. (1989). Implications of studies of expertise in pedagogy for teacher education and evaluation. New directions for teacher assessment. Princenton: Educational Testing Service.

BerLiner, D. C. (1990). At-risk and expert teachers: some thoughts about their evaluation. Journal of Personnel Evaluation, 4, 75-90.

CASTELó, A. (1986). Bases per a la realització d'un programa de recerca sobre la identificació d'individus superdotats/ben dotats, com a dades fonamentals per l'estudi psicopedagògic de la e'xcepcionalitat. Bellaterra: UAB (Tesis de licenciatura).

Castelló, A. (1987). La integración escolar del alumno excepcionalmente dotado. En Monereo (Comp.). Areas de intervención del psicólogo de la educación en la integración escolar del alumno con necesidades educativas especiales. Barcelona: ECOM.

Centra, J. A. (1983). Research productivity and teacher effectiveness. Research in Higher Education, 18, 379-389.

CHI. M.T.H.; GLASER, R. \& FARR. M. (1988). The nature of expertise. Hillsdale: Lawrence Erlbaum. 
EISNER, E. W. (1986). Research on teaching the visual arts. En Wittrock, M. C. (Ed.). Handbook of research on teaching. New York: Macmillan.

Fernández Sánchez, J. (1988). La evaluación de la enseñanza universitaria: la experiencia de la Complutense. Studia Pedagogica, 20, 135-146.

Gage, N. L. Y Berliner, D.C. (1984). Educational Psychology. Boston: Houghton Mifflin.

Gehlbach, R.D. (1990). Art education: issues in curriculum and research. Educational Researcher, 19, $7,19-25$.

Genovard, C. (1982). Hacia un esquema previo para el estudio del superdotado. Cuadernos de Psicología, 6(1), $115-144$.

Genovard, C. (1983A). Educación especial para profesores de educación especial de niños excepcionales superdotados: inventando el futuro. Educar, 4, 101-109.

Genovard, C. (1983b). Dades complementàries per a una revisió dels diferents tipus d'excepcionalitat en els subjectes superdotats. En Primer simpòsium nacional sobre psicopedagogia del l'excepcionalitat. Barcelona: Edicions Caixa de Pensions.

Genovard, C. (1990). Estudio preliminar sobre la identificación del alumno superdotado. Madrid: Fundación Juan March.

Genovard, C \& Castello, A. (1990). El limite superior. Aspectos psicopedagógicos de la excepcionalidad intelectual. Madrid: Pirámide.

Genovard, C \& Gotzens, C. (1990). Psicología de la instrucción. Madrid: Santillana.

Genovard, C; Gotzens, C.; Castelló, A.; González, C. \& Gonzdilez, J. P. (en prensa). Conceptualización, metodologá y modelización cognitiva de la evaluación de la docencia universitaria: la experiencia de la Universidad Autónoma de Barcelona. Bellaterra: ICE UAB.

Genovard, C.; Gotzens, C. \& Montane, J. (1987). Psicología de la Educación. Una nueva perspectiva interdisciplinaria. Barcelona: CEAC.

Klausmeier, H. J. (1985). Educational Psychology. New York: Harper and Row.

MAYER, R. E. (1981). The promise of cognitive psychology. New York: W. H. Freeman \& Co. (Traducción al castellano: el futuro de la psicología cognitiva. Madrid: Alianza Editorial).

Parsons, M. J. (1987). How we understand art. A cognitive developmental account of aesthetic experience. Cambridge: Cambridge University Press.

Renzulli.J. S. (1984). The triad/revolving door system: a research-based approach to identification and programming for the gifted and talented. Gifted Child Quarterly, 28, 4, 163-171.

Salvador, L. \& Sanz, J. J. (1988). Evaluación de la docencia mediante cuestionario de alumnos: Universidad de Cantabria (curso 86-87). Studia Pedagogica, 20, 41-71.

Terman. L. M. (1925). Mental and phisical traits of thousand gifted cbildren. Genetic studies of genius. Standford: Standford University Press.

\section{Extended Summary}

Four lines of research related to the assessment of cognitive processes are discussed in this paper. Their primary aim is to improve instructional performance. Although cognitive variables are not frequently used as explanatory tools in instructional studies, they offer a wide framework for describing, analyzing, and interpreting instructional processes.

These four lines of research could be organized according to their location on a continuum: going from a theoretical to an applied dimension. The former dimension includes explanatory and descriptive functions, while the latter includes practical and professional activities. Another, perhaps more obvious, organization criterium could be based on who performs the cognitive processing: teacher or student, who represent two of the main instructional variables.

In relation to the teacher, and from a theoretical perspective, the work on expert teaching is described. A cognitive processual model (MCR) has been developed based on David Berliner's work. From an applied perspective, the role of the teacher as a detector of gifted and talented students is studied together with its implications for teaching strategies.

In relation to the student, and from a theoretical perspetive, we discuss research work on cognitive abilities involved in high-technological enviroments. These are increasingly present in our lives and in the educational context. In terms of applied research, there is the work on factual understanding of aesthetic aptitudes in people involves in artistic education. 